\title{
Asma en pediatría: claves para su diagnóstico y tratamiento según evidencia basada en el paciente
}

\author{
Miles Weinberger MD' \\ I Professor of Pediatrics. Pediatric Allergy \& Pulmonary Division. University of lowa Children’s Hospital.
}

\section{PATIENT-ORIENTED EVIDENCE BASED ESSENTIALS THAT MATTERS FOR MANAGEMENT OF CHILDREN WITH ASTHMA}

Asthma is one of the most prevalent chronic diseases in childhood. Diagnosis is still controversial in young patients. Misdiagnoses will delay the start of treatment. Several phenotypes are described, but these vary during the course of the disease, or can coexist. This article describes the key points to achieve an accurate clinical diagnosis, characteristic phenotypes, and basic therapeutic strategies.

Key words: Asthma, phenotypes, therapy.

\section{RESUMEN}

Siendo el asma una de las enfermedades crónicas de mayor prevalencia en pediatría, su diagnóstico aún es controversial en los pacientes de corta edad. En ocasiones este se confunde, lo que posterga el inicio del tratamiento adecuado. Se han descrito diversos fenotipos, sin embargo, estos pueden variar durante la evolución de la enfermedad, o pueden coexistir. Se describen los puntos esenciales para lograr un diagnóstico clínico certero, los fenotipos característicos, y las estrategias terapéuticas básicas.

Palabras clave: Asma, fenotipos, tratamiento.

\section{INTRODUCCIÓN}

El asma es el diagnóstico médico más común entre los niños hospitalizados. En los Estados Unidos, el asma da cuenta cerca del 15\% de los ingresos no-quirúrgicos en pediatría. Además es de las causas más importantes de consultas de urgencia. También es causante de ausentismo escolar, morbilidad considerable, discapacidad y mortalidad ocasional a cualquier edad('). A pesar de estas estadísticas desalentadoras, existe información que indica que la falla en el manejo del asma no siempre es el resultado de una terapia inadecuada sino que corresponde a una atención médica inadecuada ${ }^{(2,3)}$.

\section{DIAGNÓSTICO DE ASMA}

El diagnóstico del asma en el niño pequeño es controvertido( ${ }^{(4)}$. Con frecuencia las exacerbaciones se diagnostican erróneamente como bronquitis o neumonía, o como "enfermedad reactiva de la vía aérea”(5). El asma se define como

\section{Correspondencia:}

Miles Weinberger MD

E-mail: contacto@neumologia-pediatrica.cl

Versión original en inglés. Tema presentado por Dr. Miles Weinberger en el IX Congreso de la Sociedad Chilena de Neumología Pediátrica, 2013, y revisión solicitada para su publicación en la revista Neumología Pediátrica

Traducción y resumen: Dra. Solange Caussade. una enfermedad caracterizada por una hiperreactividad de la vía aérea frente a diversos estímulos, lo que resulta en una obstrucción, la cual es reversible de manera espontánea o como resultado del tratamiento. La obstrucción de la vía aérea es resultado de niveles variables de broncoespasmo e inflamación. La inflamación produce edema de la mucosa y secreción de mucus.

Debe considerarse el diagnóstico de asma en los pacientes que presentan alguno de los siguientes síntomas:

- Sibilancias recurrentes o crónicas especialmente espiratorias.

- Tos recurrente o crónica.

- Diagnóstico frecuente de bronquitis.

- Diagnóstico frecuente de neumonía, clínicamente no concordantes con la presencia de infección bacteriana.

El diagnóstico de asma se confirma demostrando la respuesta clínica (remisión completa de síntomas), o la reversibilidad de la obstrucción bronquial en la espirometría luego de inhalación de $\beta_{2}$ agonista, o luego de la administración de corticosteroides en altas dosis por 5 a 10 días.

\section{CARACTERIZACIÓN CLÍNICA DEL ASMA}

\section{Patrones fenotípicos}

Ya que el asma es de naturaleza hetereogénea, no basta solamente diagnosticarla para poder desarrollar el plan de tratamiento más adecuado. La planificación de estrategias eficientes y eficaces para el manejo del asma requiere la iden- 
tificación del patrón clínico de cada paciente. Los patrones clínicos o fenotipos pueden ser generalmente identificados con una breve historia clínica preguntando en forma dirigida los siguientes antecedentes:

- Edad de inicio de los síntomas respiratorios bajos.

- Los síntomas de asma isólo se asocian a síntomas de infección respiratoria viral?

- Entre los episodios de síntomas respiratorios, iel niño presenta tos o sibilancias?

- ¿Existe alguna variación estacional de los síntomas? iLa estación corresponde a aquella en la que es posible la inhalación de alérgenos o a la estación en la que aumentan las infecciones respiratorias virales?

- Los síntomas respiratorios iestán relacionados a exposiciones ambientales específicas?

- Los síntomas respiratorios bajos ise presentan en forma diaria por períodos prolongados?

A partir de las respuestas a estas preguntas, se puede determinar el patrón clínico de asma:

Intermitente: Este patrón se caracteriza por síntomas episódicos. El fenotipo más común son aquellos pacientes en los cuales el asma es solamente gatillado por una infección viral respiratoria con períodos completamente asintomáticos entre estas infecciones virales. De forma típica los padres dirán "cada vez que mi hijo/hija se resfría, el resfrío se le va al pecho". Entre cada resfrío, estos pacientes no presentan ningún signo de inflamación de la vía aérea cuando se les estudia mediante lavado broncoalveolar durante los períodos asintomáticos $^{(6)}$.

Crónico: Los pacientes con asma crónica experimentan virtualmente síntomas diarios durante todo el año $y$, en ausencia de terapia de mantención adecuada, no tienen períodos extensos sin síntomas. Estos niños podrían empezar con un patrón intermitente inducido por infecciones virales, y luego evolucionar hacia síntomas diarios persistentes. La mayoría, pero no todos, tienen un componente alérgico.

Alérgico estacionario: Estos pacientes experimentan síntomas diarios virtuales durante los períodos estacionales de aeroalergenos.

\section{SEVERIDAD}

Todos los patrones clínicos mencionados pueden variar en severidad. Pueden ser desde muy leves hasta de riesgo vital. Las preguntas para evaluar la severidad son:

- Los síntomas respiratorios iinterfieren con el sueño?

- Los síntomas respiratorios zinterfieren con las actividades diarias?

- Frecuencia de uso de medicación de rescate con broncodilatador y corticosteroides sistémicos.

- Frecuencia de consulta ambulatoria o en servicio de urgencia.

- Frecuencia de hospitalización.

- Requerimiento de cuidado intensivo.

- Requerimiento de asistencia ventilatoria.

- Eventos de aparente amenaza a la vida.

\section{TRATAMIENTO DEL ASMA}

El tratamiento del asma se puede dividir en dos estrategias terapéuticas: las medidas de intervención en caso de síntomas agudos y las medidas de mantención para la prevención de síntomas futuros.

\section{Tratamiento de intervención}

Los $\beta_{2}$ agonistas inhalados son la primera medida de intervención para síntomas agudos. Aunque producen un rápido inicio de la broncodilatación, los $\beta_{2}$ agonistas no alteran el componente inflamatorio del asma que contribuye a la obstrucción de las vías respiratorias causando edema mucoso y secreción de mucus.

Como los $\beta_{2}$ agonistas no alteran el componente inflamatorio de la obstrucción de la vía aérea, la terapia anti-inflamatoria adquiere un rol esencial. La inhalación de corticosteroides, incluso en altas dosis, resultó no ser eficaz para las exacerbaciones agudas de asma ${ }^{(7)}$, las cuales son en su mayoría causadas por infecciones de las vías respiratorias ${ }^{(8)}$. Contrario al poco o casi nulo efecto de la inhalación de corticosteroides sobre las exacerbaciones agudas de asma, múltiples estudios han demostrado que el uso agresivo precoz de esteroides sistémicos proporciona un gran beneficio clínico en niños que cursan una exacerbación aguda de asma ${ }^{(9-13)}$.

La administración precoz de corticosteroides sistémicos para el asma agudo permite el alta precoz ${ }^{(9)}$, disminuye la posibilidad de hospitalización de pacientes que consultan en urgencia ${ }^{(10,11)}$, y evita la progresión de exacerbaciones de asma en pacientes ambulatorios que están en riesgo de necesitar atención de urgencia ${ }^{(12)}$. La administración oral de corticosteroides se mantiene como promedio 5 a 7 días y no debe exceder los 10 días ${ }^{(14-16)}$. Además, la administración pre$\mathrm{coz}$ de corticosteroides orales durante los primeros síntomas de una infección viral respiratoria previene la evolución hacia un asma agudo severo en niños con un historial que indica un alto riesgo de requerir hospitalización $n^{(13)}$.

El lugar más adecuado para tratar síntomas agudos de asma es donde éstos ocurran, en la casa, en el colegio o donde el niño esté jugando. El tratamiento en la consulta, en la sala de emergencias, o en el hospital deberían ser generalmente considerados como manifestación de falla del tratamiento. De hecho, la medida más eficaz para tratar el asma agudo son los medicamentos inhalados y orales. Estas medidas son más eficaces si se usan antes de necesitar consulta médica de urgencia.

\section{Tratamiento de mantención}

El tratamiento de mantención está indicado en los pacientes con asma crónica y en aquellos con asma alérgico estacional prolongado. Debe utilizarse medicamentos seguros en forma diaria, que supriman los síntomas asmáticos y mantengan una función pulmonar normal. Los corticosteroides inhalados son el tratamiento de mantención más eficaz. Es mejor agregar un broncodilatador de acción prolongada (LABA) que suministrar una dosis alta de corticoesteroides ${ }^{(17,18)}$. Sin embargo, han aparecido estudios que muestran tolerancia al efecto broncoprotector de $\beta_{2}$ agonistas de acción prolongada debido a la estimulación continua de receptores $\beta_{2}$ adrenérgicos ${ }^{(19,20)}$. 
Un pequeño subconjunto de pacientes presentaría riesgo de efectos adversos por LABA. Se ha asociada un leve aumento de mortalidad en pacientes asmáticos con la administración de $L A B A^{(21,22)}$. Se informó acerca de 2 pacientes que presentaban eventos de aparente amenaza a la vida, con pobre respuesta a broncodilatadores $\beta_{2}$ agonistas, quienes recibían salmeterol; al suspender este medicamento mostraron una respuesta clínica dramática ${ }^{(23)}$. Esto es consistente con estudios que mostraron que un cierto polimorfismo genético del receptor de $\beta_{2}$ estaba asociado a una regulación negativa de este receptor durante la administración regular de agentes $\beta_{2}$ agonistas ${ }^{(24-28)}$. La teofilina es un medicamento suplementario alternativo de eficacia similar a un LABA, a pesar que su uso es a menos conveniente(29).

\section{CONCLUSIONES}

El asma y sus enfermedades relacionadas, la rinitis y la dermatitis atópica, son problemas médicos que requieren considerar la información basada en la evidencia, concentrándose en las medidas que tendrían con mayor probabilidad un mayor impacto. El manejo de estas enfermedades requiere la adherencia a las indicaciones médicas, educación del paciente, evaluación regular minuciosa y en especial la comprensión de su enfermedad por parte del paciente. De esta forma es posible obtener resultados exitosos.

\section{REFERENCIAS}

I. Mannino DM, Homa DM, Akinbami LJ, Moorman JE, Gwynn C, Redd SC. Surveillance for asthma-United States, 1980-1999. MMWR Surveill Summ 2002; 51 : 1 - 13.

2. Kelly CS, Morrow AL, Shults J, Nakas N, Strope GL, Adelman RD. Outcomes evaluation of a comprehensive intervention program for asthmatic children enrolled in Medicaid. Pediatrics 2000; 105: 1029-35.

3. Najada A, Abu-Hasan M, Weinberger M. Outcome of asthma in children and adolescents at a specialty based care program. Ann Allergy Asthma Immunol 200 I; 87: 335-43.

4. Weinberger M, Abu-Hasan M. Asthma in preschool children. In Kendig's Disorders of the Respiratory Tract in Children, 7th Edition, Saunders Elsevier, Philadelphia 2006; p 795-807.

5. Fahy JV, O'Byrne PM. Reactive airways disease. A lazy term of uncertain meaning that should be abandoned. Am J Respir Crit Care Med 2000; 163: 822-3.

6. Maclennan C, Hutchinson P, Holdsworth S, Bardin PG, Freezer NJ. Airway inflammation in asymptomatic children with episodic wheeze. Pediatr Pulmonol 2006;41: 577-83.

7. Hendeles L, Sherman J. Are inhaled corticosteroid effective for acute exacerbations of asthma in children? J Pediatr 2003; 142: S26-S33.

8. Lemanske RF. Viruses and asthma: inception, exacerbation, and possible prevention. J Pediatr 2003; I42: S3-S8.

9. Storr J, Barrell E, Barry W, Lenney W, Hatcher G. Effect of a single oral dose of prednisolone in acute childhood asthma. Lancet 1987; I: 879-82.

10. Tal A, Levy N, Bearman JE. Methylprednisolone therapy for acute asthma in infants and toddlers: a controlled clinical trial. Pediatrics 1990; 86: 350-6.

I I. Scarfone RJ, Fuchs SM, Nager AL, Shane SA. Controlled trial of oral prednisone in the emergency department treatment of children with acute asthma. Pediatr 1993; 92: 513-8.

12. Harris JB, Weinberger M, Nassif E, Smith G, Milavetz G, Stillerman A. Early intervention with short courses of prednisone to prevent progression of asthma in ambulatory patients incompletely responsive to bronchodilators. J Pediatr 1987; I 10: 627-44.

13. Brunette MG, Lands L, Thibodeau LP. Childhood asthma: prevention of attacks with short-term corticosteroid treatment of upper respiratory tract infection. Pediatr 1988; 81: 624-9.

14. Lederle FA Pluhar RE Joseph AM Niewoehner DE. Tapering of corticosteroid therapy following exacerbation of asthma. A randomized, double-blind, placebo-controlled trial. Arch Intern Med 1987; 147: 2201-3.

15. O'Driscoll BR Kalra S Wilson M Pickering CA Carroll KB Woodcock AA. Double-blind trial of steroid tapering in acute asthma. Lancet 1993; 341: 324-7.

16. Karan RS, Pandhi P, Behera D, Saily R, Bhargava VK. A comparison of non-tapering vs tapering prednisolone in acute exacerbation of asthma involving use of the low-dose ACTH test. Int J Clin Pharmacol Ther 2002; 40: 256-62.

17. Greening AP, Ind P, Northfield M, Shaw G. Added salmeterol versus higher-dose corticosteroid in asthma patients with symptoms on existing inhaled corticosteroid (Allen \& Hanburys Limited UK Study Group). Lancet 1994; 344: 219-324.

18. Woolcock A, Lundback B, Ringdal N, Jacques LA. Comparison of addition of salmeterol to inhaled steroids with doubling of the dose of inhaled steroid. Am J Respir Crit Care Med 1996; I53: I48I-8.

19. Anderson SD, Caillaud C, Brannan JD. $\beta_{2}$ agonists and exerciseinduced asthma. Clin Rev Allergy Immunol 2006; 31: 163-80.

20. Haney S, Hancox RJ. Recovery from bronchoconstriction and bronchodilator tolerance. Clin Rev Allergy Immunol 2006; 31 : 181-96.

21. Castle W, Fuller R, Hall J, Palmer J. Serevent nationwide surveillance study: Comparison of salmeterol with salbutamol in asthmatic patients who require regular bronchodilator treatment. BMJ 1993; 306: 1034-7.

22. Nelson HS, Weiss ST, Bleecker ER, Yancey SW, Dorinsky PM, and the SMART Study Group. The salmeterol multicenter asthma research trial: a comparison of usual pharmacotherapy for asthma or usual pharmacotherapy plus salmeterol. Chest 2006; 129: 5-26.

23. Weinberger M, Abu-Hasan M. Life threatening asthma during treatment with salmeterol. N Engl J Med 2006; 335: 852-3.

24. Israel E, Drazen JM, Liggett SB, et al. The effect of polymorphism of the beta2-adrenergic receptor on the response to regular use of albuterol in asthma. Am J Respir Crit Care Med 2000; I62: 75-80.

25. Lee DK, Currie GP, Hall IP, Lima JJ, Lipworth BJ. The arginine- 16 beta2-adrenoceptor polymorphism predisposes to bronchoprotective subsensitivity in patients treated with formoterol and salmeterol. Br J Clin Pharmacol 2004; 57: 68-75.

26. Israel E, Chinchilli VM, Ford JG, et al. Use of regularly scheduled albuterol treatment in asthma: genotype-stratified, randomized, placebo-controlled cross-over trial. Lancet 2004; 364: I 505- 12.

27. Wechsler ME, Lehman E, Lazarus SC, et al. $\beta$-adrenergic receptor polymorphisms and response to salmeterol. Am J Respir Crit Care Med 2006; 173: 519-26.

28. Palmer CNA, Lipworth BJ, Ismail T, Macgregor DF, Mukhopadhyay S. Arginine-16 $\beta_{2}$ adrenoceptor genotye predisposes to exacerbations in young asthmatics taking regular salmeterol. Thorax 2006; 61 : 940-4.

29. Weinberger $M$, Hendeles L. Theophylline in asthma. N Engl J Med 1996; 334: 1380-8. 